\title{
Assessing the Mental Health of Brazilian Students Involved in Risky Behaviors
}

\author{
Daise Fernanda Santos Souza Escobar ${ }^{1}$, Priscilla Rayanne e Silva Noll 1,2 ${ }^{\mathbb{B}}$, \\ Thaís Ferreira de Jesus ${ }^{1}$ and Matias Noll ${ }^{1, * \text { (D }}$ \\ 1 Public Health, Instituto Federal Goiano-Campus Ceres, Ceres 76300-000, Brazil; \\ daise.ifgoianoceres@gmail.com (D.F.S.S.E.); priscilla.silva@ifgoiano.edu.br (P.R.eS.N.); \\ thaisferreiradejesus@hotmail.com (T.F.d.J.) \\ 2 Department of Obstetrics and Gynecology, Faculdade de Medicina, Universidade de São Paulo, \\ São Paulo 05508-070, Brazil \\ * Correspondence: matias.noll@ifgoiano.edu.br; Tel.: +55-62-9-8125-3657
}

Received: 25 April 2020; Accepted: 20 May 2020; Published: 22 May 2020

\begin{abstract}
Adolescence, which is the transition from childhood to adulthood, is marked by emotional sensitivity and inconsistency and may be affected by mental health problems. In order to fill the gap related to the risky behaviors in students in Brazil, our cross-sectional study aimed to analyze the relationship between risky behaviors and indicators of mental health of Brazilian students. We used the data from the National School Health Survey to analyze the relationship between risk behaviors and three symptoms of mental health issues: feeling of being alone, number of close friends, and trouble sleeping due to worries. The sample consisted of 102,072 students in Brazil (48.3\% boys and $51.7 \%$ girls), aged between 11 to 19 years. The risk behaviors evaluated were substance use, sedentary lifestyle, sexual behavior, and suffering violence and bullying. We have performed a multivariate analysis based on the Poisson regression model, and the measure of effect used was the prevalence ratio (PR) with confidence intervals (CI) of 95\%. Our results showed that students with symptoms of mental health issues were involved in risky behaviors, including drug use and unsafe sex. Thus, mental illness outcomes may be associated with risky behaviors, or mental health may be impaired by them. Given these findings, in-school programs focused on improving mental health outcomes should be developed.
\end{abstract}

Keywords: adolescents; risky behaviors; school; substance abuse; mental health

\section{Introduction}

Mental health is a subject of extreme social importance [1-4]. For individuals with low socioeconomic status, poor mental health outcomes may also lead to further issues in their daily lives [1,4-6]. To address this, countries such as the United States, the United Kingdom, and France have invested resources into their mental health systems [7]. In Brazil, with the use of the World Health Organization (WHO) guidelines and the creation of The Mental Health Policy in 2001, increased mental health services have begun to allow discussions around the subject, as well as provide better treatment conditions [7]. However, although many improvements in the field of mental health have been achieved, the relevant amount of population studies conducted in Brazil are insufficient when compared to that of other developed countries, indicating a need for more research focused on mental health [8]. Furthermore, as of 2001, more than $90 \%$ of countries globally did not include adolescents in their mental health policies, even though this age group is often most affected by mental health disorders [1].

During adolescence, individuals experience developmental changes [9-11]. The transition from childhood to adulthood is marked by emotional sensitivity and inconsistency, which can lead to 
intensified feelings $[2,9,12]$. This can also contribute to mental health disorders and interfere with positive decision-making skills $[10,13]$. Hence, episodes of rebellion and dangerous habits, such as substance abuse and violent behavior, may start to develop [9,11,13].

A study [14] conducted with 2227 students in grades six through eight in North Carolina, United States, demonstrated that substance use was associated with attempting to be included and accepted by their peers and other members of social groups. Hence, the poor state of mental health was considered a factor that could lead to drug use. Other studies $[10,13,15]$ conducted on adolescents have shown similar results that link substance use with poor mental health outcomes. Addiction has also been linked to poor mental health outcomes, including the feeling of being alone and trouble sleeping, especially when the substances, such as nicotine or alcohol, can create a chemical dependence $[16,17]$.

Alcohol is the most accessible illicit substance in Brazil. It is common for Brazilian adolescents to have access to both alcoholic beverages and cigarettes, despite laws that prohibit the sale of these products to minors [16]. Although Brazil has public policies aimed at preventing smoking and alcohol use, alcohol and tobacco are still widely consumed by adults and adolescents, especially by those who experience social vulnerability and by children whose parents smoke [10]. The same applies to alcohol use; children of alcohol-dependent parents are more susceptible to becoming alcohol-dependent too [18]. Furthermore, alcohol is a substance considered socially acceptable in Brazil, even among minors. In addition, drug use and other risky behaviors can increase the risk of poor mental health outcomes [10]. There is also an association between alcohol consumption and smoking cigarettes, along with other drugs [10,19], unsafe sexual practices [20], and violent behaviors [10].

Being a victim of physical aggression or of bullying [21] is related to poor mental health results in many communities, including the ones in Brazil [14,20]. Unfortunately, it is also common for young people with mental health disorders to engage in premature sexual activity and participate in other harmful activities in an attempt to escape their problems [10]. In addition to the personal impact, social consequences are also associated with these risky behaviors; low professional productivity, behavioral problems, and the economic cost of healthcare paid by the country and families continue from adolescence to adulthood [22,23]. Other problems are school-related damages, such as poor grades, dropouts, bullying and its consequences [20,24], sedentarism, [25] and risky sexual behaviors [20]. We realize it is necessary to understand whether there is an association between mental health and the adoption of risky behaviors in students in Brazil.

Adolescents spend a lot of time in school and establish a significant amount of their interpersonal relationships in this environment. Therefore, schools are an important data source [21]. Thus, our study aimed to analyze the relationship between risky behaviors and three indicators of mental health of Brazilian students. Our specific objectives were to verify the association between the aforementioned three mental health indicators and (a) substance use, (b) sexual behavior, (c) sedentarism, (d) suffered bullying, and (e) suffered physical violence.

We have evaluated mental health through three indicators: (a) feeling alone, (b) lacking close friends, and (c) having trouble sleeping due to worries. As our study is a populational study, we used these three variables as indicators of mental health [20], not as psychiatric disorder exams. According to WHO [26], these indicators are fundamental for the promotion of mental health, which is related to the capacity for a healthy personal and social relationship. Therefore, we do not diagnose the mental health of adolescents; we only check for the presence of these symptoms, which are related to insomnia and loneliness and indicate poor mental health outcomes [1,27]. We have hypothesized that risky behaviors, such as frequent use of cigarettes, drugs and alcohol consumption, risky sexual behavior, sedentarism, and suffered bullying, are higher indicators of poor mental health. 


\section{Materials and Methods}

\subsection{Type of Study}

We conducted a cross-sectional study using data from the National School Health Survey (PeNSE) database, a survey conducted in partnership between the Brazilian Institute of Geography and Statistics (https://www.ibge.gov.br/) and the ministries of health (http://www.saude.gov.br) and of education (http://portal.mec.gov.br/). In 2015, the PeNSE was approved by the National Health Council and by the National Commission of Ethics in Research (CONEP, protocol n. 1.006.467 of 30.03.2015), which regularizes and approves Brazilian research involving human beings [28].

\subsection{Population}

The PeNSE sample was dimensioned to infer population parameters of the geographic domains analyzed. These domains included the five major geographical regions in Brazil (North, Northeast, Midwest, Southeast, and South), as well as the 26 Federative Units of Brazil and the Federal District, including capitals and municipalities. The municipal samples and the geographical strata of the capitals were obtained randomly and with equal probability and were calculated with the following parameters: a prevalence ratio of 0.5 , a maximum error of $0.03 \%$, and a confidence interval of $95 \%$. A class sample was obtained for each school and an independent sample of students for each stratum. The sample consisted of 120,122 students from 4159 classes of 3040 schools. Sample loss, owing to the total number of absent students, was $14.8 \%$. The final sample included 102,072 students who were present on the day of data collection and participated in the study [28].

\subsection{Data Collection and Analysis Procedures}

The data collection for the version of the PeNSE used in this study occurred between April and September 2015. The PeNSE used self-report questionnaires delivered to students by using smartphones. The technicians distributed the smartphones to students present in the classroom on the day of data collection and explained how to use the device. All students in the sampled classes were invited to answer the questionnaires, and those who agreed to participate completed an informed consent form (ICF). PeNSE data collection assesses various health and quality of life outcomes, and several studies are dedicated to analyzing these aspects based on their results [29-33].

As it is a population-based survey, PeNSE evaluated broad issues related to insomnia and loneliness $[27,34,35]$ through behaviors such as feeling alone, having no more than one close friend, and difficulty sleeping $[12,27]$. Then, indicators of mental health were assessed using the following objective-type questions (Table 1):

Table 1. Questions used to access the indicators of mental health.

\begin{tabular}{ll}
\hline \multicolumn{1}{c}{ Questions } & \multicolumn{1}{c}{ Possible Answer Options } \\
\hline In the last 12 months, how often have you felt alone? & $\begin{array}{l}\text { (1) Never or rarely } \\
\text { (2) Sometimes } \\
\text { (3) Most often or always }\end{array}$ \\
\hline $\begin{array}{ll}\text { In the last 12 months, how often did you not get to sleep at } \\
\text { night because something was worrying you a lot? }\end{array}$ & $\begin{array}{l}\text { (1) Never or rarely } \\
\text { (2) Sometimes } \\
\text { (3) Most often or always }\end{array}$ \\
\hline How many close friends do you have? & (1) Up to one friend \\
& (2) Two friends or more \\
\hline
\end{tabular}

These questions were asked because, although such events may occur many times during a lifetime, when combined and recurrent, they are signs of mental illness [36]. We also evaluated sociodemographic characteristics (gender, age, and mother's education). 
According to the definition of risk behaviors [37], the following risky behavioral factors were considered to be exposure variables (Table 2):

Table 2. Questions used to access risky behavioral factors.

\begin{tabular}{|c|c|}
\hline Risky Behavioral Factors & Specific Assessment Questions \\
\hline Use of cigarettes & $\begin{array}{l}\text { Have you ever smoked cigarettes, even a puff or two? } \\
\text { In the last } 30 \text { days, on how many days did you smoke cigarettes? }\end{array}$ \\
\hline Alcohol consumption & $\begin{array}{l}\text { Have you consumed alcoholic beverages during your lifetime? } \\
\text { In the last } 30 \text { days, on how many days did you drink } \\
\text { alcoholic beverages? } \\
\text { Did you drink until you became drunk? }\end{array}$ \\
\hline Use illicit drugs & $\begin{array}{l}\text { Have you ever used illicit drugs in your lifetime? } \\
\text { In the last } 30 \text { days, on how many days did you use illicit drugs? }\end{array}$ \\
\hline Age at onset of sexual activity and condom use & $\begin{array}{l}\text { How old were you when you engaged in your first act of } \\
\text { sexual intercourse? } \\
\text { Did you use a condom during your first act of sexual intercourse? }\end{array}$ \\
\hline Being bullied and/or experiencing aggression & $\begin{array}{l}\text { Have you ever suffered bullying? } \\
\text { How many times were you physically attacked in the last } \\
12 \text { months? }\end{array}$ \\
\hline Sedentarism & On a normal day, how many hours do you spend sitting? \\
\hline
\end{tabular}

The data was analyzed using descriptive statistics and the Wald chi-square association test (bivariate analysis) for the three variables regarding mental health. The following risk behavioral factors were considered independent variables: use of cigarettes and illicit drugs, alcohol consumption, age at onset of sexual activity, condom use, incidence of being bullied and/or experiencing aggression, and sedentarism. Independent variables with a significance level of $p<0.2$ in the bivariate analysis were included in an adjusted analysis with sociodemographic variables (gender, age, and mother's level of education) through the Poisson regression model with robust variance. The measure effect was the prevalence ratio (PR) ( $\alpha=0.05)$. The statistical analyses were performed using the Statistical Package for the Social Sciences (SPSS 23) (IBM, Armonk, NY, USA).

\section{Results}

In total, 102,072 students answered the questionnaire—of which, 49,290 were male and 52,782 were female. Our results indicated that female adolescents feel more alone (55.9\%), are more likely to have no more than one friend $(10.7 \%)$, and have more difficulties falling asleep due to worries $(45.1 \%)$ than male adolescents $(32.7 \%, 10.3 \%$, and $26.7 \%$, respectively) (Table 3$)$. With respect to age, our results revealed that the older students were more likely to feel alone $(47.0 \%)$, have trouble sleeping due to worries (41.6\%), and have fewer friends (13.9\%) (Table 3). The mother's level of education influenced all the variables, indicating that the lower the education level of their maternal figures, the more likely students are to feel alone ( $47.6 \%)$, have fewer friends (14.6\%), and have trouble sleeping due to more frequent worries $(41.8 \%)$ (Table 3$)$.

The results of the bivariate analysis indicated that mental health indicators are associated with smoking cigarettes, drinking alcoholic beverages, drug use (Table 4), early sexual intercourse, no use of condoms (Table 5), sedentary activity, and experiencing aggression and bullying (Table 6). In the adjusted analysis (Table 7), the feeling of being alone was associated with being physically assaulted four times or more in the last 12 months ( $P R=1.56, C I=1.52-1.59$ ). For students who did not have more than one close friend, there was an association with the use of cigarettes (PR $=1.24, \mathrm{CI}=1.18-1.20)$ and illicit drugs $(\mathrm{PR}=1.26, \mathrm{CI}=1.18-1.35)$ at least once in the student's lifetime. Alcohol experimentation was not significant in the adjusted analysis $(\mathrm{PR}=0.783)$. 
Table 3. Sample description of the present study and prevalence of a loneliness feeling, number of close friends, and insomnia due to worries among 9th grade adolescents in the 2015 PeNSE $(N=102,072)$.

\begin{tabular}{|c|c|c|c|c|c|c|c|c|c|c|c|}
\hline \multirow[t]{2}{*}{ Variables } & \multicolumn{2}{|c|}{ Total } & \multicolumn{3}{|c|}{ Feel Alone } & \multicolumn{3}{|c|}{$\begin{array}{l}\text { Does Not Have More } \\
\text { Than } 1 \text { Close Friend }\end{array}$} & \multicolumn{3}{|c|}{$\begin{array}{c}\text { Have Trouble Sleeping } \\
\text { Due to Worries }\end{array}$} \\
\hline & $\mathbf{N}$ & $\%$ & $\mathbf{N}$ & $\%$ & $p$ & $\mathbf{N}$ & $\%$ & $p$ & $\mathbf{N}$ & $\%$ & $p$ \\
\hline \multicolumn{12}{|l|}{ Gender } \\
\hline Male & 49,290 & 48.3 & 16,035 & 32.7 & $<0.001$ & 5026 & 10.3 & 0.016 & 13,062 & 26.7 & $<0.001$ \\
\hline Female & 52,782 & 51.7 & 29,427 & 55.9 & & 5620 & 10.7 & & 23,731 & 45.1 & \\
\hline \multicolumn{12}{|l|}{ Age } \\
\hline$\leq 13$ y.o. & 17,260 & 16.9 & 7487 & 43.5 & \multirow{4}{*}{$<0.001$} & 1488 & 8.6 & \multirow{4}{*}{$<0.001$} & 5666 & 32.9 & \multirow{4}{*}{$<0.001$} \\
\hline 14 y.o. & 51,611 & 50.6 & 22,839 & 44.4 & & 5010 & 9.7 & & 18,070 & 35.1 & \\
\hline 15 y.o. & 20,864 & 20.4 & 9382 & 45.2 & & 2452 & 11.8 & & 7978 & 38.5 & \\
\hline 16 y.o. or more & 12,337 & 12.1 & 5754 & 47.0 & & 1696 & 13.9 & & 5079 & 41.6 & \\
\hline \multicolumn{12}{|l|}{ Mother's Education } \\
\hline Did not study & 5531 & 5.4 & 2622 & 47.6 & \multirow{4}{*}{$<0.001$} & 802 & 14.6 & \multirow{4}{*}{$<0.001$} & 2297 & 41.8 & \multirow{4}{*}{$<0.001$} \\
\hline Started primary education & 24,241 & 23.7 & 11,075 & 45.8 & & 2748 & 11.4 & & 9009 & 37.3 & \\
\hline Started secondary school & 24,178 & 23.7 & 10,811 & 44.8 & & 2421 & 10.0 & & 8577 & 35.6 & \\
\hline Started post-secondary school & 22,688 & 22.2 & 10,036 & 44.3 & & 1897 & 8.4 & & 7902 & 34.9 & \\
\hline
\end{tabular}

PeNSE: Pesquisa Nacional de Saúde do Escolar (National School Health Survey).

Sedentarism had an inverse relationship with the possibility of having close friends for students who commonly sit for more than six hours a day ( $P R=0.89, \mathrm{CI}=0.84-0.95)$. That is, students who stay seated longer have more friends. Not having more than one close friend was associated with bullying ( $\mathrm{PR}=1.37, \mathrm{CI}=1.31-1.43)$ and being physically assaulted four times or more in the last 12 months ( $\mathrm{PR}=1.70, \mathrm{CI}=1.58-1.82)$. Having trouble sleeping due to worries was also associated with using cigarettes $(\mathrm{PR}=1.37, \mathrm{CI}=1.34-1.39)$, alcohol $(\mathrm{PR}=1.40, \mathrm{CI}=1.37-1.42)$, or illicit drugs ( $\mathrm{PR}=1.38, \mathrm{CI}=1.35-1.42)$. Remaining seated for six hours or more per day ( $\mathrm{PR}=1.24, \mathrm{CI}=1.20-1.27)$, not using condoms in the first act of sexual intercourse ( $P R=1.12, C I=1.09-1.16)$, bullying $(P R=1.44$, $\mathrm{CI}=1.41-1.47)$, and being assaulted four times or more in the last 12 months $(\mathrm{PR}=1.60, \mathrm{CI}=1.55-1.65)$ were also associated with not sleeping due to worries (Table 7).

The students who indicated they experienced the symptoms assessed for with the mental health questionnaire had a higher frequency of cigarette, alcohol, and illicit drug use, ranging from three or more days in the last 30 days. Onset of sexual activity prior to age 15 was inversely related to the feeling of being alone and not having more than one close friend (Table 7). 
Table 4. Results of bivariate analysis between substance use and mental health indicators among 9th grade adolescents in the 2015 PeNSE.

\begin{tabular}{|c|c|c|c|c|c|c|c|c|c|}
\hline \multirow{2}{*}{ Variables } & \multicolumn{3}{|c|}{ Feel Alone } & \multicolumn{3}{|c|}{ Does Not Have More than 1 Close Friend } & \multicolumn{3}{|c|}{ Have Trouble Sleeping Due to Worries } \\
\hline & $\%$ & PR & $p$ & $\%$ & PR & $p$ & $\%$ & PR & $p$ \\
\hline \multicolumn{10}{|l|}{ Smoked cigarettes } \\
\hline No & 42.4 & 1 & \multirow{2}{*}{$<0.001$} & 9.9 & 1 & \multirow{2}{*}{$<0.001$} & 33.9 & 1 & \multirow{2}{*}{$<0.001$} \\
\hline Yes & 54.9 & $1.30(1.28-1.32)$ & & 12.9 & $\begin{array}{c}1.31 \\
(1.25-1.36)\end{array}$ & & 46.6 & $\begin{array}{c}1.38 \\
(1.35-1.40)\end{array}$ & \\
\hline \multicolumn{10}{|c|}{ In the last 30 days, how many days did you smoke cigarettes } \\
\hline None & 54.2 & 1 & \multirow{4}{*}{$<0.001$} & 12.7 & 1 & \multirow{4}{*}{$<0.001$} & 44.4 & 1 & \multirow{4}{*}{$<0.001$} \\
\hline 1 to 2 days & 56.6 & $1.04(1.01-1.02)$ & & 12.4 & $\begin{array}{c}0.98 \\
(0.88-1.09)\end{array}$ & & 51.0 & $\begin{array}{l}1.15 \\
(1.10-1.20)\end{array}$ & \\
\hline 3 to 9 days & 59.8 & $1.10(1.05-1.16)$ & & 12.2 & $\begin{array}{c}0.96 \\
(0.83-1.12)\end{array}$ & & 54.1 & $\begin{array}{c}1.22 \\
(1.16-1.29)\end{array}$ & \\
\hline 10 or more & 54.8 & $1.01(0.96-1.07)$ & & 17.8 & $\begin{array}{c}1.41 \\
(1.24-1.60)\end{array}$ & & 52.8 & $\begin{array}{c}1.19 \\
(1.13-1.26)\end{array}$ & \\
\hline \multicolumn{10}{|c|}{$\begin{array}{l}\text { Have you been consumed alcoholic beverages during } \\
\text { your lifetime }\end{array}$} \\
\hline No & 38.2 & 1 & \multirow{2}{*}{$<0.001$} & 10.3 & 1 & \multirow{2}{*}{0.049} & 29.5 & 1 & \multirow{2}{*}{$<0.001$} \\
\hline Yes & 50.8 & $1.33(1.31-1.35)$ & & 10.7 & $\begin{array}{c}1.04 \\
(1.00-1.06)\end{array}$ & & 42.4 & $\begin{array}{c}1.44 \\
(1.41-1.46)\end{array}$ & \\
\hline \multicolumn{10}{|c|}{$\begin{array}{l}\text { In the last } 30 \text { days, how many days did you drink } \\
\text { alcoholic beverages }\end{array}$} \\
\hline None & 48.5 & 1 & \multirow{4}{*}{$<0.001$} & 10.9 & 1 & \multirow{4}{*}{$<0.001$} & 38.4 & 1 & \multirow{4}{*}{$<0.001$} \\
\hline 1 to 2 days & 52.8 & $1.09(1.07-1.11)$ & & 10.2 & $\begin{array}{c}0.94 \\
(0.88-0.99)\end{array}$ & & 45.6 & $\begin{array}{c}1.19 \\
(1.16-1.22)\end{array}$ & \\
\hline 3 to 9 days & 54.0 & $1.11(1.08-1.14)$ & & 10.0 & $\begin{array}{c}0.011 \\
0.9199)\end{array}$ & & 48.9 & 1.27 & \\
\hline 10 or more & 53.5 & $1.10(1.06-1.14)$ & & 12.6 & $\begin{array}{c}0.84-0.999 \\
1.16 \\
(1.05-1.29)\end{array}$ & & 53.9 & $\begin{array}{c}(1.24-1.31) \\
1.40 \\
(1.35-1.46)\end{array}$ & \\
\hline \multirow{2}{*}{\multicolumn{10}{|c|}{$\begin{array}{l}\text { Did you drink until you became drunk } \\
\text { None }\end{array}$}} \\
\hline & 47.9 & 1 & \multirow{4}{*}{$<0.001$} & 10.2 & 1 & \multirow{4}{*}{$<0.001$} & 38.4 & 1 & \multirow{4}{*}{$<0.001$} \\
\hline 1 to 2 days & 55.0 & $1.15(1.13-1.17)$ & & 11.2 & $\begin{array}{c}1.09 \\
(1.03-1.16)\end{array}$ & & 46.9 & $\begin{array}{c}1.22 \\
(1.20-1.25)\end{array}$ & \\
\hline 3 to 9 days & 55.0 & $1.15(1.12-1.18)$ & & 12.0 & $\begin{array}{c}1.18 \\
(1.08-1.28)\end{array}$ & & 51.3 & $\begin{array}{c}1.34 \\
(1.30-1.38)\end{array}$ & \\
\hline 10 or more & 52.8 & $1.10(1.06-1.15)$ & & 12.5 & $\begin{array}{c}1.23 \\
(1.09-1.37)\end{array}$ & & 51.6 & $\begin{array}{c}1.34 \\
(1.29-1.40)\end{array}$ & \\
\hline \multicolumn{10}{|c|}{ Used illicit drugs in your lifetime } \\
\hline No & 43.8 & 1 & \multirow{2}{*}{$<0.001$} & 10.2 & 1 & \multirow{2}{*}{$<0.001$} & 35.0 & 1 & \multirow{2}{*}{$<0.001$} \\
\hline Yes & 54.8 & $1.26(1.23-1.28)$ & & 13.5 & $\begin{array}{c}1.32 \\
(1.25-1.40)\end{array}$ & & 49.0 & $\begin{array}{c}1.40 \\
(1.37-1.43)\end{array}$ & \\
\hline \multicolumn{10}{|c|}{ In the last 30 days, how many days did you use illicit drugs } \\
\hline None & 53.7 & 1 & & 13.6 & 1 & & 47.0 & 1 & \\
\hline 1 to 2 days & 57.2 & $1.07(1.02-1.12)$ & 0.023 & 12.2 & $\begin{array}{c}0.90 \\
(0.78-1.03)\end{array}$ & 0.002 & 49.8 & $\begin{array}{c}1.06 \\
(1.01-1.12)\end{array}$ & $<0.001$ \\
\hline 3 to 9 days & 56.6 & $1.05(0.99-1.12)$ & & 12.5 & $\begin{array}{c}0.92 \\
(0.78-1.10)\end{array}$ & & 54.4 & $\begin{array}{c}1.16 \\
(1.09-1.23)\end{array}$ & \\
\hline 10 or more & 52.9 & $0.98(0.92-1.06)$ & & 17.4 & $\begin{array}{c}1.28 \\
(1.08-1.51)\end{array}$ & & 51.3 & $\begin{array}{c}1.09 \\
(1.02-1.18)\end{array}$ & \\
\hline
\end{tabular}

PeNSE: Pesquisa Nacional de Saúde do Escolar (National School Health Survey), PR: prevalence ratio, and CI: confidence interval. 
Table 5. Results of bivariate analysis between sexual behavior and mental health indicators of bivariate analysis among 9th grade adolescents in the 2015 PeNSE.

\begin{tabular}{|c|c|c|c|c|c|c|c|c|c|}
\hline \multirow{2}{*}{ Variables } & \multicolumn{3}{|c|}{ Feel Alone } & \multicolumn{3}{|c|}{ Does Not Have More Than 1 Close Friend } & \multicolumn{3}{|c|}{ Have Trouble Sleeping Due to Worries } \\
\hline & $\%$ & PR & $p$ & $\%$ & PR & $p$ & $\%$ & PR & $p$ \\
\hline 15 y.o. or more & 48.9 & 1 & \multirow{2}{*}{$<0.001$} & 13.8 & 1 & \multirow{2}{*}{$<0.001$} & 44.5 & 1 & \multirow{2}{*}{$<0.001$} \\
\hline 12 y.o. or less & 42.7 & $\begin{array}{c}0.87 \\
(0.84-0.91) \\
\end{array}$ & & 13.0 & $\begin{array}{c}0.94 \\
(0.86-1.03)\end{array}$ & & 36.1 & $\begin{array}{c}0.81 \\
(0.79-0.85)\end{array}$ & \\
\hline \multicolumn{10}{|c|}{$\begin{array}{l}\text { Use of condom during first act of } \\
\text { sexual intercourse }\end{array}$} \\
\hline No & 50.9 & $\begin{array}{c}1.16 \\
(1.13-1.19)\end{array}$ & $<0.001$ & 14.2 & $\begin{array}{c}1.26 \\
(1.18-1.34)\end{array}$ & $<0.001$ & 44.6 & $\begin{array}{c}1.13 \\
(1.09-1.16)\end{array}$ & $<0.001$ \\
\hline
\end{tabular}

PeNSE: Pesquisa Nacional de Saúde do Escolar (National School Health Survey), PR: prevalence ratio;, and CI: confidence interval.

Table 6. Results of bivariate analysis risk behaviors and mental health indicators of bivariate analysis among 9th grade adolescents in the 2015 PeNSE.

\begin{tabular}{|c|c|c|c|c|c|c|c|c|c|}
\hline \multirow{2}{*}{ Variables } & \multicolumn{3}{|c|}{ Feel Alone } & \multicolumn{3}{|c|}{ Does Not Have More Than 1 Close Friend } & \multicolumn{3}{|c|}{ Have Trouble Sleeping Due to Worries } \\
\hline & $\%$ & PR & $p$ & $\%$ & PR & $p$ & $\%$ & PR & $p$ \\
\hline \multicolumn{10}{|c|}{$\begin{array}{l}\text { In an average day, how many hours do } \\
\text { you spend sitting }\end{array}$} \\
\hline Up to $1 \mathrm{~h}$ & 40.2 & 1 & \multirow{4}{*}{$<0.001$} & 13.0 & 1 & \multirow{4}{*}{$<0.001$} & 34.7 & 1 & \\
\hline 1 to $3 \mathrm{~h}$ & 40.8 & $\begin{array}{c}1.02 \\
(0.99-1.04)\end{array}$ & & 9.9 & $\begin{array}{c}0.76 \\
(0.73-0.81)\end{array}$ & & 32.7 & $\begin{array}{c}0.94 \\
(0.92-0.97)\end{array}$ & \multirow[t]{3}{*}{$<0.001$} \\
\hline 3 to $6 \mathrm{~h}$ & 45.1 & $\begin{array}{c}1.12 \\
(1.10-1.15)\end{array}$ & & 9.3 & $\begin{array}{c}0.72 \\
0.68-0.75)\end{array}$ & & 35.4 & $\begin{array}{c}(0.92-0.9 /) \\
1.02 \\
(1.00-1.05)\end{array}$ & \\
\hline$>6 \mathrm{~h}$ & 52.3 & $\begin{array}{c}1.30 \\
(1.28-1.33)\end{array}$ & & 10.4 & $\begin{array}{c}0.80 \\
(0.76-0.85)\end{array}$ & & 42.3 & $\begin{array}{c}1.22 \\
(1.19-1.25)\end{array}$ & \\
\hline \multicolumn{10}{|c|}{ Have you ever suffered bullying } \\
\hline No & 34.8 & 1 & \multirow{2}{*}{$<0.001$} & 8.9 & 1 & \multirow[t]{2}{*}{$<0.001$} & 29.5 & 1 & \multirow{2}{*}{$<0.001$} \\
\hline Yes & 55.3 & $\begin{array}{c}1.59 \\
(1.57-1.61)\end{array}$ & & 12.0 & $\begin{array}{c}1.34 \\
(1.29-1.39)\end{array}$ & & 43.2 & $\begin{array}{c}1.47 \\
(1.44-1.49)\end{array}$ & \\
\hline \multicolumn{10}{|c|}{$\begin{array}{l}\text { How many times were you physically } \\
\text { attacked in the last } 12 \text { months }\end{array}$} \\
\hline None & 41.3 & 1 & \multirow{4}{*}{$<0.001$} & 9.9 & 1 & \multirow{4}{*}{$<0.001$} & 33.3 & 1 & \multirow{4}{*}{$<0.001$} \\
\hline Once & 58.2 & $\begin{array}{c}1.41 \\
(1.38-1.42)\end{array}$ & & 11.7 & $\begin{array}{c}1.19 \\
(1.11-1.26)\end{array}$ & & 47.2 & $\begin{array}{c}1.42 \\
(1.38-1.45)\end{array}$ & \\
\hline 2 to 3 times & 62.2 & $\begin{array}{c}1.51 \\
(1.47-1.54)\end{array}$ & & 12.3 & $\begin{array}{c}1.24 \\
(1.15-1.35)\end{array}$ & & 50.6 & $\begin{array}{c}1.52 \\
(1.47-1.56)\end{array}$ & \\
\hline 4 or more times & 62.0 & $\begin{array}{c}1.50 \\
(1.47-1.53)\end{array}$ & & 15.7 & $\begin{array}{c}1.59 \\
(1.49-1.69)\end{array}$ & & 51.8 & $\begin{array}{c}1.55 \\
(1.51-1.60)\end{array}$ & \\
\hline
\end{tabular}

PeNSE: Pesquisa Nacional de Saúde do Escolar (National School Health Survey), PR: prevalence ratio, and CI: confidence interval. 
Table 7. Multivariate analysis adjusted by gender, age, and mother's education.

\begin{tabular}{|c|c|c|c|c|c|c|}
\hline \multirow{2}{*}{ Variables } & \multicolumn{2}{|c|}{ Feel Alone } & \multicolumn{2}{|c|}{$\begin{array}{l}\text { Does Not Have More Than } 1 \\
\text { Close Friend }\end{array}$} & \multicolumn{2}{|c|}{$\begin{array}{l}\text { Have Trouble Sleeping Due } \\
\text { to Worries }\end{array}$} \\
\hline & PR Adjusted & $p$ & PR Adjusted & $p$ & PR Adjusted & $p$ \\
\hline \multicolumn{7}{|c|}{ Have you smoked cigarettes during your lifetime } \\
\hline No & 1 & \multirow{2}{*}{$<0.001$} & 1 & \multirow{2}{*}{$<0.001$} & 1 & \multirow{2}{*}{$<0.001$} \\
\hline Yes & $1.31(1.29-1.33)$ & & $1.24(1.18-1.20)$ & & $1.37(1.34-1.39)$ & \\
\hline \multicolumn{7}{|c|}{ In the last 30 days, how many cigarettes did you smoke } \\
\hline None & 1 & \multirow{4}{*}{$<0.001$} & 1 & \multirow{4}{*}{$<0.001$} & 1 & \multirow{4}{*}{$<0.001$} \\
\hline 1 to 2 days & $1.02(0.98-1.06)$ & & $0.94(0.83-1.07)$ & & $1.08(1.03-1.13)$ & \\
\hline 3 to 9 days & $1.11(1.06-1.17)$ & & $0.94(0.78-1.12)$ & & $1.22(1.15-1.30)$ & \\
\hline 10 or more & $1.05(0.99-1.12)$ & & $1.47(1.27-1.70)$ & & $1.24(1.17-1.33)$ & \\
\hline \multicolumn{7}{|c|}{ Have you been consumed alcohol during your lifetime } \\
\hline No & 1 & \multirow{2}{*}{$<0.001$} & 1 & \multirow{2}{*}{0.783} & 1 & \multirow{2}{*}{$<0.001$} \\
\hline Yes & $1.31(1.29-1.33)$ & & $1.01(0.6-1.05)$ & & $1.40(1.37-1.42)$ & \\
\hline \multicolumn{7}{|c|}{$\begin{array}{l}\text { In the last } 30 \text { days, how many days did you drink } \\
\text { alcoholic beverages }\end{array}$} \\
\hline None & 1 & \multirow{4}{*}{$<0.001$} & 1 & \multirow{4}{*}{$<0.001$} & 1 & \multirow{4}{*}{$<0.001$} \\
\hline 1 to 2 days & $1.06(1.04-1.08)$ & & $0.91(0.85-0.98)$ & & $1.14(1.11-1.17)$ & \\
\hline 3 to 9 days & $1.11(1.08-1.14)$ & & $0.90(0.82-0.99)$ & & $1.27(1.23-1.31)$ & \\
\hline 10 or more & $1.13(1.08-1.17)$ & & $1.16(1.04-1.31)$ & & $1.41(1.35-1.47)$ & \\
\hline \multicolumn{7}{|c|}{ Did you drink until drunk } \\
\hline None & 1 & \multirow{4}{*}{$<0.001$} & 1 & \multirow{4}{*}{$<0.001$} & 1 & \multirow{4}{*}{$<0.001$} \\
\hline 1 to 2 days & $1.14(1.11-1.16)$ & & $1.06(0.99-1.14)$ & & $1.20(1.18-1.24)$ & \\
\hline 3 to 9 days & $1.17(1.13-1.21)$ & & $1.16(1.06-1.28)$ & & $1.36(1.31-1.40)$ & \\
\hline 10 or more & $1.17(1.12-1.23)$ & & $1.17(1.02-1.34)$ & & $1.42(1.35-1.49)$ & \\
\hline \multicolumn{7}{|c|}{ Have you been used illicit drugs during your lifetime } \\
\hline No & 1 & \multirow{2}{*}{$<0.001$} & 1 & \multirow{2}{*}{$<0.001$} & 1 & 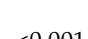 \\
\hline Yes & $1.26(1.23-1.29)$ & & $1.26(1.18-1.35)$ & & $1.38(1.35-1.42)$ & $<0.001$ \\
\hline In the last 30 days & & & & & & \\
\hline None & 1 & & 1 & & 1 & \\
\hline 1 to 2 days & $1.05(1.00-1.10)$ & & $0.89(0.76-1.04)$ & & $1.05(0.99-1.11)$ & \\
\hline 3 to 9 days & $1.05(0.99-1.12)$ & 0.195 & $0.97(0.80-1.17)$ & 0.003 & $1.16(1.09-1.25)$ & $<0.001$ \\
\hline 10 or more & $1.04(0.96-1.13)$ & & $1.34(1.11-1.62)$ & & $1.20(1.11-1.30)$ & \\
\hline In a normal day, $h$ & & & & & & \\
\hline Up to $1 \mathrm{~h}$ & 1 & & 1 & & 1 & \\
\hline 1 to $3 \mathrm{~h}$ & $1.02(0.99-1.05)$ & 0001 & $0.81(0.76-0.86)$ & O 001 & $0.97(0.94-1.00)$ & - 0001 \\
\hline 3 to $6 \mathrm{~h}$ & $1.13(1.11-1.16$ & $<0.001$ & $0.79(0.75-0.84)$ & $<0.001$ & $1.07(1.04-1.10)$ & $<0.001$ \\
\hline $6 \mathrm{~h}$ or more & $1.29(1.26-1.32)$ & & $0.89(0.84-0.95)$ & & $1.24(1.20-1.27)$ & \\
\hline How old were you & & & & & & \\
\hline
\end{tabular}


Table 7. Cont

\begin{tabular}{|c|c|c|c|c|c|c|}
\hline \multirow{2}{*}{ Variables } & \multicolumn{2}{|c|}{ Feel Alone } & \multicolumn{2}{|c|}{$\begin{array}{l}\text { Does Not Have More Than } 1 \\
\text { Close Friend }\end{array}$} & \multicolumn{2}{|c|}{$\begin{array}{l}\text { Have Trouble Sleeping Due } \\
\text { to Worries }\end{array}$} \\
\hline & PR Adjusted & $p$ & PR Adjusted & $p$ & PR Adjusted & $p$ \\
\hline 15 years or more & 1 & \multirow{3}{*}{$<0.001$} & 1 & \multirow{3}{*}{$<0.001$} & 1 & \multirow{3}{*}{0.509} \\
\hline 13 and 14 years & $0.92(0.89-0.96)$ & & $0.79(0.72-0.86)$ & & $0.98(0.94-1.02)$ & \\
\hline 12 years or less & $0.91(0.87-0.96)$ & & $0.85(0.76-0.96)$ & & $0.97(0.92-1.03)$ & \\
\hline \multicolumn{7}{|c|}{ Use of condom in first act of sexual intercourse } \\
\hline Yes & 1 & \multirow[b]{2}{*}{$<0.001$} & 1 & \multirow{3}{*}{$<0.001$} & 1 & \multirow[b]{2}{*}{$<0.001$} \\
\hline No & $1.15(1.11-1.18)$ & & $1.26(1.17-1.36)$ & & $1.12(1.09-1.16)$ & \\
\hline \multicolumn{6}{|l|}{ Suffered bullying } & \\
\hline No & 1 & \multirow{2}{*}{$<0.001$} & 1 & \multirow{2}{*}{$<0.001$} & 1 & \multirow{2}{*}{$<0.001$} \\
\hline Yes & $1.56(1.54-1.59)$ & & $1.37(1.31-1.43)$ & & $1.44(1.41-1.47)$ & \\
\hline \multicolumn{7}{|c|}{$\begin{array}{l}\text { How many times were you physically attacked in the last } 12 \\
\text { months }\end{array}$} \\
\hline None & 1 & \multirow{4}{*}{$<0.001$} & 1 & \multirow{4}{*}{$<0.001$} & 1 & \\
\hline Once & $1.41(1.38-1.44)$ & & $1.25(1.16-1.34)$ & & $1.40(1.36-1.44)$ & \multirow{3}{*}{$<0.001$} \\
\hline 2 to 3 times & $1.50(1.46-1.54)$ & & $1.29(1.17-1.41)$ & & $1.54(1.49-1.59)$ & \\
\hline 4 or more times & $1.56(1.52-1.59)$ & & $1.70(1.58-1.82)$ & & $1.60(1.55-1.65)$ & \\
\hline
\end{tabular}

PeNSE: Pesquisa Nacional de Saúde do Escolar (National School Health Survey), PR: prevalence ratio, and CI: confidence interval 


\section{Discussion}

Mental health outcomes can be affected by many factors [23]. Determining the mental state of an individual is a meticulous process that requires preparation, complex evaluation, and the use of appropriate and individual examination instruments [1]. Hence, the PeNSE does not provide enough data to evaluate the mental health of students. However, it does present three indicators that assess the feeling of being alone, lack of close friendships, and trouble sleeping due to worries, and the frequency of these experiences may suggest poor mental health outcomes. Though these three factors are normal human experiences and can occur several times throughout one's life, if they recur frequently, they should not be disregarded as common occurrences, as they may indicate symptoms of mental health disorders. The number of close friends one has is another indicator. As people are naturally social, not having more than one close friend can be considered as a symptom of isolation and difficulty with socialization $[38,39]$. The feeling of being alone can also be a symptom presented by socially excluded adolescents, and the two-way relationship between exclusion and mental health has already been established [40]. Sleep quality is also an important factor for mental health [35], and to have trouble sleeping is associated with mental illness [34] and suicidal ideation [21]. Thus, it should be considered that an adolescent who frequently presents with two or more of these symptoms may experience poor mental health outcomes. Mental health symptoms do not necessarily result in the development of severe clinical pathology, but they can badly impact one's quality of life [20,21].

These three variables of mental health indicators were associated with experimentation (used at least once in a lifetime) of cigarettes, alcohol, and/or illicit drugs. The relationship between substance use and mental health indicators can be reciprocal; that is, the use of drugs can lead to poor mental health outcomes or the opposite [22]. During adolescence, mental health concerns combined with impulsiveness and the need to belong to a group may influence substance use [11].

Our study indicates that the frequency of drug use was similarly related to mental health indicators. The findings show that not having more than one close friend has been related to adolescents' alcohol consumption and cigarette smoking on ten or more of the last 30 days. Moderate consumption (during one to nine of the last 30 days), however, had a direct association with the number of close friends; that is, adolescents indulging in moderate alcohol consumption had more than one close friend. Therefore, our results agreed with our research objectives, indicating that use of these substances may increase social inclusion for adolescents, explaining why adolescent substance users have more close friends. However, consuming alcohol on ten or more out of 30 days may be related to the development of alcohol dependence. Furthermore, drinking alcohol to the point of intoxication on ten or more days in the last 30 days was related to the three indicators assessed for in our study. A study conducted in 2018 revealed similar results [38], and more frequent alcohol use has been shown to be associated with social isolation [41].

Additionally, our findings showed that using illicit drugs on ten or more out of the last 30 days was correlated with having no more than one friend and experiencing trouble sleeping due to worries. These results are critical, because, in addition to poor mental health outcomes, heavy alcohol use can have harmful medical consequences as well [41,42]. For example, prior research has noted that heavy alcohol use can lead to memory impairment [43].

Poor mental health outcomes can be the consequence or the cause of substance use and vice versa, affecting both the individual and society $[10,13]$. For adolescents, substance use can alter neurodevelopment, creating aggressive behaviors and possibly leading to engagement in criminal activities. Moreover, their personal lives, including schooling, can be impaired. Mental health disorders can lead to dropping out of school, and even if the individual graduates with a basic education, he/she will generally make less income over a lifetime than those who do not experience poor mental health. Therefore, in addition to the harms the individual may experience as an adolescent, he or she may also experience a lower socioeconomic status as an adult. Usually, the expenses incurred are related to mental health and substance abuse treatment, and while they can be paid for by the individual's family, they may also impact the state. Furthermore, substance use has been associated with the reduction of 
labor, increased crime, poverty, unemployment, and early mortality, as well as the development of mental health disorders such as depression and schizophrenia [22]. In addition, substance use has been associated with other physical health concerns such as obesity [44].

Regarding adolescent sexual behaviors, our findings showed that early onset of sexual activity was inversely related to feeling alone and having only one close friend; most likely because, when they are in relationships, adolescents establish bonds of affection. Additionally, the findings of our study were in agreement with other research findings indicating that, although adolescents are aware of the risks from engaging in unprotected sexual intercourse, many still choose not to use condoms [45,46]. Furthermore, prior research has indicated that the use of condoms during the first act of sexual intercourse favors the regularity of the behavior [46]. Some factors may increase the occurrence of nonusage of condoms, such as drug use and mental health disorders [45]. Unprotected sexual intercourse may result in the transmission of sexually transmitted infections and/or adolescent pregnancy, both of which have been shown to increase the likelihood of poor mental health outcomes, such as depression [47].

Not engaging in regular physical activity is also a harmful behavior; mental health and sedentarism have a reciprocal relationship; one shows influence on the other and vice versa. [25]. In our study, we noted that, generally, sitting for six hours or more in a day is associated with feeling alone and experiencing trouble sleeping due to worries. However, our findings also showed that being sedentary was positively associated with having more friends. It is possible that, while sedentary, adolescents engage in activities such as using a computer, playing videogames, and/or social networking, establishing friendships in the process [48].

Our study also found that being a victim of bullying and being physically assaulted was also associated with not having more than one close friend, feeling alone, and having trouble sleeping due to worries. Another study, carried out from 2014 to 2015, analyzed students from Vietnam and identified an association between experiencing bullying and depressive symptoms and suicidal ideation [24]. Aggressive behavior has also been reported as a behavioral change indicative of a mental illness manifestation [49]. Violence and victimization have been shown to be related to poor mental health outcomes as well [50].

Our study has some limitations. First, our findings do not infer causality. Moreover, these datasets are based on self-reports, and the questionnaire was developed specifically for this Brazilian National Survey. The findings may be influenced by social self-perception. It is important to note that PeNSE did not measure psychiatric disorders. We evaluated indicators of mental health from issues related to insomnia and loneliness. Though our survey data was collected in 2015, it is the most recent population survey representative of Brazilian adolescents. Despite the limitations, our analysis showed that the three studied variables ("feeling alone", "not having more than one close friend", and "to have trouble sleeping due to worries"), which are indicative of poor mental health outcomes, are related to suggested risky behaviors (substance use, not using a condom during the first act of sexual intercourse, leading a sedentary lifestyle, and being a victim of physical violence and bullying). In this sense, these variables that are related to other public health problems and impact the mental health not only of adolescents but of their family nuclei need to be further investigated. It is also important to understand the role of the school and the family in promoting a healthy environment for mental health. Consequently, it is valid to say that Brazilian students need actions that are focused on discussing poor mental health outcomes and risk behaviors, because these problems are present in the population and are related to each other.

\section{Conclusions}

Drug use causes many problems, mainly related to crime. Being the target of violence and sedentarism can also cause harm to the subject and entail many expenses related to clinical care. Not using condoms during sex can lead to other problems, such as sexually transmitted infections and unplanned pregnancy. In our study, these behaviors were associated with at least one mental illness symptom. Therefore, it is suggested that further studies are developed to understand more aspects 
of this relationship between risk behaviors and mental health. We also suggest that interdisciplinary initiatives be implemented in schools. An interesting proposal is to talk about mental health in the classroom and present data on risky behaviors and their consequences. After all, an adolescent is less likely to start using drugs or not worry about his mental health if he knows that his psychological and cognitive functions can be adversely affected and, perhaps, permanently affected. So, we hope this study will inspire new studies and intervention actions to help the Brazilian context.

Author Contributions: Conceptualization, D.F.S.S.E. and M.N.; methodology, P.R.eS.N. and M.N.; software, D.F.S.S.E., T.F.d.J., and M.N.; validation, D.F.S.S.E., P.R.eS.N., T.F.d.J., and M.N.; formal analysis, P.R.eS.N. and M.N.; investigation, D.F.S.S.E., P.R.eS.N., T.F.d.J., and M.N.; resources, D.F.S.S.E., P.R.eS.N., T.F.d.J., and M.N.; data curation, D.F.S.S.E., P.R.eS.N., T.F.d.J., and M.N.; writing—original draft preparation, D.F.S.S.E.; writing—review and editing, D.F.S.S.E., P.R.eS.N., T.F.d.J., and M.N.; visualization, P.R.eS.N. and M.N.; supervision, P.R.eS.N. and M.N.; and project administration, P.R.eS.N. and M.N. All authors have read and agreed to the published version of the manuscript.

Funding: This research received no external funding.

Acknowledgments: We thank the Federal Institute of Goiano (Instituto Federal Goiano) for support.

Conflicts of Interest: The authors declare no conflict of interest.

\section{References}

1. World Health Organization. World Health Report; WHO: Genebra, Switzerland, 2001; ISBN 972-675-082-2.

2. Bowman, S.; McKinstry, C.; Howie, L.; McGorry, P. Expanding the search for emerging mental ill health to safeguard student potential and vocational success in high school: A narrative review. Early Interv. Psychiatry 2020, 14, eip.12928. [CrossRef] [PubMed]

3. WHO. Depression and Other Common Mental Disorders. Available online: https://www.who.int/mental_ health/management/depression/prevalence_global_health_estimates/en/ (accessed on 21 May 2020).

4. Amaral, C.E.; Onocko-Campos, R.; De Oliveira, P.R.S.; Pereira, M.B.; Ricci, É.C.; Pequeno, M.L.; Emerich, B.; Dos Santos, R.C.; Thornicroft, G. Systematic review of pathways to mental health care in Brazil: Narrative synthesis of quantitative and qualitative studies. Int. J. Ment. Health Syst. 2018, 12, 1-14. [CrossRef] [PubMed]

5. Biswas, T.; Scott, J.G.; Munir, K.; Thomas, H.J.; Huda, M.M.; Hasan, M.M.; David de Vries, T.; Baxter, J.; Mamun, A.A. Global variation in the prevalence of bullying victimisation amongst adolescents: Role of peer and parental supports. EClinicalMedicine 2020, 20, 100276. [CrossRef] [PubMed]

6. Pryor, L.; Lioret, S.; van der Waerden, J.; Fombonne, É.; Falissard, B.; Melchior, M. Food insecurity and mental health problems among a community sample of young adults. Soc. Psychiatry Psychiatr. Epidemiol. 2016, 51 , 1073-1081. [CrossRef] [PubMed]

7. World Health Organization. Mental Health; WHO: Belo Horizonte, Brazil, 2018; ISBN 9788589737678.

8. De Araújo, T.M.; De Palma, T.F.; Do Araújo, N.C. Work-related Mental Health Surveillance in Brazil: Characteristics, difficulties, and challenges. Cien. Saude Colet. 2017, 22, 3235-3246. [CrossRef]

9. Thorsen, M.L.; Pearce-Morris, J. Adolescent Mental Health and Dating in Young Adulthood. Soc. Ment. Health 2016, 6, 223-245. [CrossRef]

10. Ferreira, V.R.; Jardim, T.V.; Sousa, A.L.L.; Rosa, B.M.C.; Jardim, P.C.V. Smoking, alcohol consumption and mental health: Data from the Brazilian study of Cardiovascular Risks in Adolescents (ERICA). Addict. Behav. Rep. 2018, 9, 100147. [CrossRef]

11. Volkow, N.D.; Koob, G.F.; Croyle, R.T.; Bianchi, D.W.; Gordon, J.A.; Koroshetz, W.J.; Pérez-Stable, E.J.; Riley, W.T.; Bloch, M.H.; Conway, K.; et al. The conception of the ABCD study: From substance use to a broad NIH collaboration. Dev. Cogn. Neurosci. 2018, 32, 4-7. [CrossRef]

12. World Health Organization. Growing Up Unequal: Gender and Socioeconomic Differences in Young People's Health and Well-Being; Europe, W.H.O.: Copenhagen, Denmark, 2016.

13. Claro, H.G.; Oliveira, M.A.F.D.; Titus, J.C.; Fernandes, I.F.D.A.L.; Pinho, P.H.; Tarifa, R.R. Drug use, mental health and problems related to crime and violence: Cross-sectional study. Rev. Lat. Am. Enferm. 2015, 23, 1173-1180. [CrossRef] 
14. DuRant, R.H.; Smith, J.A.; Kreiter, S.R.; Krowchuk, D.P. The Relationship Between Early Age of Onset of Initial Substance Use and the Clustering of Health Risk Behaviors Among Young Adolescents • 5. Pediatr. Res. 1998, 43, 3. [CrossRef]

15. Meredith, L.S.; Ewing, B.A.; Stein, B.D.; Shadel, W.G.; Holliday, S.B.; Parast, L.; Amico, E.J.D. Influence of mental health and alcohol or other drug use risk on adolescent reported care received in primary care settings. BCM Fam. Pract. 2018, 19, 1-9. [CrossRef] [PubMed]

16. Vieira, D.L.; Ribeiro, M.; Laranjeira, R. Evidence of association between early alcohol use and risk of later problems. Rev. Bras. Psiquiatr. 2007, 29, 222-227. [CrossRef] [PubMed]

17. Perry, C.L.; Jessor, R. The Concept of Health Promotion and the Prevention of Adolescent Drug Abuse. Health Educ. Q. 1985, 12, 169-184. [CrossRef] [PubMed]

18. Homel, J.; Warren, D. The Relationship Between Parent Drinking and Adolescent Drinking: Differences for Mothers and Fathers and Boys and Girls. Subst. Use Misuse 2019, 54, 661-669. [CrossRef]

19. Tapia-Rojas, C.; Carvajal, F.J.; Mira, R.G.; Arce, C.; Lerma-Cabrera, J.M.; Orellana, J.A.; Cerpa, W.; Quintanilla, R.A. Adolescent Binge Alcohol Exposure Affects the Brain Function Through Mitochondrial Impairment. Mol. Neurobiol. 2018, 55, 4473-4491. [CrossRef]

20. Mello, F.C.M.; Da Silva, J.L.; De Oliveira, W.A.; Do Prado, R.R.; Malta, D.C.; Silva, M.A.I. The practice of bullying among Brazilian schoolchildren and associated factors, National School Health Survey 2015. Cien. Saude Colet. 2017, 22, 2939-2948. [CrossRef]

21. Pfledderer, C.D.; Burns, R.D.; Brusseau, T.A. School environment, physical activity, and sleep as predictors of suicidal ideation in adolescents: Evidence from a national survey. J. Adolesc. 2019, 74, 83-90. [CrossRef]

22. Baingana, F.; Al'Absi, M.; Becker, A.E.; Pringle, B. Global research challenges and opportunities for mental health and substance-use disorders. Nature 2015, 527, S172. [CrossRef]

23. World Health Organization. Caring for Children and Adolescents with Mental Disorders; World Health Organization: Geneva, Switzerland, 2003; ISBN 9241590631.

24. Le, H.T.H.; Tran, N.; Campbell, M.A.; Gatton, M.L.; Nguyen, H.T.; Dunne, M.P. Mental health problems both precede and follow bullying among adolescents and the effects differ by gender: A cross-lagged panel analysis of school-based longitudinal data in Vietnam. Int. J. Ment. Health Syst. 2019, 13, 1-10. [CrossRef]

25. Chekroud, S.R.; Gueorguieva, R.; Zheutlin, A.B.; Paulus, M.; Krumholz, H.M.; Krystal, J.H.; Chekroud, A.M. Association between physical exercise and mental health in $1 \cdot 2$ million individuals in the USA between 2011 and 2015: A cross-sectional study. Lancet Psychiatry 2018, 5, 739-746. [CrossRef]

26. World Health Organization. Promoting Mental Health: Concepts, Emerging Evidence, Practice: Summary Report; World Health Organization: Geneva, Switzerland, 2004; ISBN 9780203987841.

27. Instituto Brasileiro de Geografia e Estatística. National School Health Survey (Pesquisa Nacional de Saúde do Escolar: 2015); IBGE: Rio de Janeiro, Brazil, 2016.

28. De Oliveira, M.M.; Campos, M.O.; De Andreazzi, M.A.R.; Malta, D.C.; De Oliveira, M.M.; Campos, M.O.; De Andreazzi, M.A.R.; Malta, D.C. Characteristics of the National Adolescent School-based Health Survey-PeNSE, Brazil. Epidemiol. Serviços Saúde 2017, 26, 605-616. [CrossRef]

29. Noll, M.; De Abreu, L.C.; Baracat, E.C.; Silveira, E.A.; Sorpreso, I.C.E. Ultra-processed food consumption by Brazilian adolescents in cafeterias and school meals. Sci. Rep. 2019, 9, 7162. [CrossRef] [PubMed]

30. Silva, R.M.A.; De Andrade, A.C.S.; Caiaffa, W.T.; De Medeiros, D.S.; Bezerra, V.M. National Adolescent School-based Health Survey-PeNSE 2015: Sedentary behavior and its correlates. PLoS ONE 2020, 15, e0228373. [CrossRef] [PubMed]

31. Costa, C.D.S.; Flores, T.R.; Wendt, A.; Neves, R.G.; Assunção, M.C.F.; Santos, I.S. Sedentary behavior and consumption of ultra-processed foods by Brazilian adolescents: Brazilian National School Health Survey (PeNSE), 2015. Cad. Saude Publica 2018, 34,1-11. [CrossRef]

32. Noll, M.; Noll, P.R.E.S.; Tiggemann, C.L.; Custodio, D.C.; Silveira, E.A. Health-risk behavior differences between boarding and non-resident students: Brazilian adolescent National School Health Survey. Arch. Public Health 2020, 78, 1-9. [CrossRef]

33. De Antunes, H.A.; Rivadeneira-Guerrero, M.F.; De Goulart, B.N.G.; Oenning, N.S.X. Familiar factors and illicit drug use among Brazilian adolescents: An analysis of the Brazilian National Survey of School Health (PeNSE, 2015). Cad. Saude Publica 2018, 34,1-11. [CrossRef]

34. Sauter, S.R.; Kim, L.P.; Jacobsen, K.H. Loneliness and friendlessness among adolescents in 25 countries in Latin America and the Caribbean. Child Adolesc. Ment. Health 2020, 25, 21-27. [CrossRef] 
35. Hsieh, Y.-P.; Lu, W.-H.; Yen, C.-F. Psychosocial Determinants of Insomnia in Adolescents: Roles of Mental Health, Behavioral Health, and Social Environment. Front. Neurosci. 2019, 13, 1-9. [CrossRef]

36. Fu, I.L.; Curatolo, E.; Friedrich, S. Affective disorders. Rev. Bras. Psiquiatr. 2000, 22, 24-27. [CrossRef]

37. Spring, B.; Moller, A.C.; Coons, M.J. Multiple health behaviours: Overview and implications. J. Public Health 2012, 34, i3-i10. [CrossRef]

38. Sheikh, M.A. Psychological abuse, substance abuse distress, dissatisfaction with friendships, and incident psychiatric problems. J. Psychosom. Res. 2018, 108, 78-84. [CrossRef] [PubMed]

39. Lima, L.B.; Pacheco, J.T.B. Symptoms, emotional self-regulation and family support: A study with children and adolescents. Estud. Interdiscip. Em Psicol. 2018, 9, 132. [CrossRef]

40. Tejerina-Arreal, M.; Parker, C.; Paget, A.; Henley, W.; Logan, S.; Emond, A.; Ford, T. Child and adolescent mental health trajectories in relation to exclusion from school from the Avon Longitudinal Study of Parents and Children. Child Adolesc. Ment. Health 2020. In press. [CrossRef]

41. Malta, D.C.; Oliveira-Campos, M.; Do Prado, R.R.; Andrade, S.S.C.; De Mello, F.C.M.; Dias, A.J.R.; Bomtempo, D.B.; Malta, D.C.; Oliveira-Campos, M.; Do Prado, R.R.; et al. Psychoactive substance use, family context and mental health among Brazilian adolescents, National Adolescent School-based Health Survey (PeNSE 2012). Rev. Bras. Epidemiol. 2014, 17, 46-61. [CrossRef] [PubMed]

42. Horta, R.L.; Horta, B.L.; Costa, A.W.N.D.; Do Prado, R.R.; Oliveira-Campos, M.; Malta, D.C. Lifetime use of illicit drugs and associated factors among Brazilian schoolchildren, National Adolescent School-based Health Survey (PeNSE 2012). Rev. Bras. Epidemiol. 2014, 17, 31-45. [CrossRef]

43. Rhodes, N.; Potocki, B.; Thomas, S. Predicting College Student Drinking and Smoking Intentions With Cognitively Accessible Attitudes and Norms. Health Educ. Behav. 2019, 46, 267-274. [CrossRef]

44. Pelletier, J.E.; Lytle, L.A.; Laska, M.N. Stress, Health Risk Behaviors, and Weight Status Among Community College Students. Health Educ. Behav. 2016, 43, 139-144. [CrossRef]

45. Tolera, F.H.; Girma, E.; Mamo, A.; Babure, Z.K. Risky sexual behaviors and associated factors among high and preparatory school youth, East Wollega, Ethiopia, 2017: A cross-sectional study design. J. Public Health Epidemiol. 2019, 11, 1-12. [CrossRef]

46. De Moura, L.R.; Torres, L.M.; Cadete, M.M.M.; De Cunha, C.F. Factors associated with health risk behaviors among Brazilian adolescents: An integrative review. Rev. Da Esc. Enferm. Da USP 2018, 52, 1-11. [CrossRef]

47. Xavier, C.G.; Brown, H.K.; Benoit, A.C. Teenage pregnancy and long-term mental health outcomes among Indigenous women in Canada. Arch. Womens. Ment. Health 2018, 21, 333-340. [CrossRef]

48. Reboussin, B.A.; Furr-Holden, D.M.; Green, K.M.; Ialongo, N.S.; Rabinowitz, J.A.; Matson, P.A.; Maher, B.; Nelson, V.; Milam, A.J. Social Influences on Drinking Trajectories From Adolescence to Young Adulthood in an Urban Minority Sample. J. Stud. Alcohol Drugs 2019, 80, 186-195. [CrossRef] [PubMed]

49. Rossetto, A.; Jorm, A.F.; Reavley, N.J. Developing a model of help giving towards people with a mental health problem: A qualitative study of Mental Health First Aid participants. Int. J. Ment. Health Syst. 2018, 12, 48. [CrossRef] [PubMed]

50. Price, M.; Hill, N.E.; Liang, B.; Perella, J. Teacher Relationships and Adolescents Experiencing Identity-Based Victimization: What Matters for Whom Among Stigmatized Adolescents. School Ment. Health 2019, 11, 790-806. [CrossRef]

(C) 2020 by the authors. Licensee MDPI, Basel, Switzerland. This article is an open access article distributed under the terms and conditions of the Creative Commons Attribution (CC BY) license (http://creativecommons.org/licenses/by/4.0/). 